\title{
ENCADENAMIENTOS PRODUCTIVOS EN COSTA RICA: UNA APLICACIÓN A PARTIR DE LOS DATOS DE LA MATRIZ INSUMO PRODUCTO
}

\author{
PRODUCTIVE CHAINS IN COSTA RICA: AN APPLICATION USING DATA FROM THE \\ INPUT-OUTPUT MATRIX
}

\section{Carlos Chaverri Morales ${ }^{1}$}

\begin{abstract}
Resumen
Uno de los principales usos que se le puede dar a la información que proporciona la matriz de insumo producto es el estudio de la estructura de producción de las economías. En particular, esta información ha resultado sumamente efectiva para identificar mediante simulaciones las actividades o productos que tienen mayor impacto en la dinámica económica. Este análisis puede contribuir al desarrollo de estrategias de política pública orientadas a impulsar el crecimiento económico y el empleo a mediano plazo.

El objetivo de este trabajo es construir y analizar los indicadores tradicionales de encadenamientos productivos hacia adelante y hacia atrás para la economía costarricense con la utilización de la información de la Matriz Insumo Producto 2011 (MIP-2011) elaborada por el Banco Central de Costa Rica. La metodología que se emplea para tales fines es la desarrollada por Hirschman (1958) y Chenery y Watanabe (1958), y que ha sido utilizada en la literatura especialmente para abordar temas relacionados con los vínculos industriales a nivel internacional.
\end{abstract}

Doi: http://dx.doi.org/10.15359/eys.21-49.2

Recibido el 14 de marzo del 2016. Reenvíos: 22 de abril de 2016, 27 de mayo de 2016. Aceptado el 30 de mayo del 2016. Publicado el 30 de mayo del 2016.

${ }^{1}$ Economista. Departamento de Investigación Económica, Banco Central de Costa Rica (BCCR), Costa Rica. Correo electrónico chaverrimc@bccr.fi.cr

Las ideas expresadas en este documento son del autor y no necesariamente representa las del Banco Central de Costa Rica.

Carlos Chaverri Morales

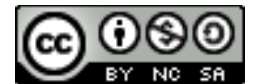

Revista Economía y Sociedad by Universidad Nacional is licensed under a Creative Commons Reconocimiento-NoComercial- 
Los resultados ratifican no solo el proceso de consolidación que ha experimentado las actividades y productos vinculadas con los servicios como motor de la actividad económica costarricense, sino además que estos generan altos encadenamientos hacia adelante.

Palabras claves: política industrial; métodos de planificación sectorial; modelo insumo-producto

\begin{abstract}
One of the main applications of the information obtained from the inputoutput matrix is the study of an economy's productive structures. This information has proven extremely effective in identifying, through simulations, activities or products that have greater impact on economic dynamics. This analysis can contribute to the development of public policy strategies aimed at boosting economic growth and employment in the medium term.
\end{abstract}

This paper is aimed at building and analyzing the traditional indicators of production chains- forwards and backwards- for the Costa Rican economy using the information from the 2011 Input Output Table (2011 OIT) prepared by the Central Bank of Costa Rica. This methodology is based on the work by Hirschman (1958) and Chenery and Watanabe (1958) and has been widely used to address various issues regarding intra-industry linkages.

Results confirm not only the consolidation process experienced by the activities and products related to the services sector as a motor of the Costa Rican economic activity, but also the idea that these activities generate the highest forward linkages.

Keywords: industrial policy; sectoral planning methods; input-output model

\title{
1. Introducción
}

Una de las principales aplicaciones del análisis de una matriz de insumo producto (en adelante MIP) es el estudio de la estructura productiva de las economías. En particular, uno de los temas más tratados ha sido la identificación de aquellas actividades o productos que tienen mayor impacto y que pueden considerarse impulsores de la actividad económica. Lo anterior constituye un elemento informativo que permite el diseño y ejecución de políticas públicas orientadas a impulsar el crecimiento económico y el empleo de mediano y largo plazo.

2

Carlos Chaverri Morales

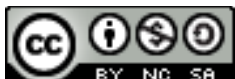

Revista Economía y Sociedad by Universidad Nacional is licensed under a Creative Commons Reconocimiento-NoComercial- 
La MIP constituye una representación simplificada de la economía que describe detalladamente el proceso de producción y la utilización de los insumos que son creados localmente, luego importados. Además, es posible cuantificar el ingreso derivado de dicha producción para cada actividad económica o producto en un periodo determinado. Por lo tanto, constituye una fuente de información que brinda los elementos necesarios para comprender las transformaciones productivas que un país experimenta y con ello disponer de datos oportunos para reorientar los estímulos económicos y crear las condiciones necesarias para fomentar la competitividad.

La información de la MIP permite, entre otras cosas, conocer el entramado productivo de la economía, así como, identificar mediante simulaciones los impactos que sufre la estructura económica como consecuencia de choques de oferta o de demanda y cómo los patrones de producción se alteran en virtud del grado de encadenamientos productivos que poseen.

En las últimas décadas la economía costarricense no estuvo aislada de los cambios productivos que experimentó la economía mundial, la mayor demanda externa por bienes y servicios además de los crecientes influjos de inversión extranjera directa (IED) han provocado cambios en la estructura de producción local, lo cual genera nuevas necesidades en términos de empleo, educación e infraestructura.

La consolidación del modelo de desarrollo vigente desde mediados de los años 80 (modelo de Promoción de Exportaciones) propició una mayor exposición de la economía al resto del mundo mediante el establecimiento de acuerdos bilaterales de comercio. El objetivo de estas políticas es generar efectos sobre el crecimiento y el empleo, principalmente por medio de una mayor difusión de las innovaciones tecnológicas, una mayor especialización y ganancia en la productividad total de los factores (PTF) y con ello la expansión de los mercados potenciales y ganancias por economías de escala.

De acuerdo con la información disponible, para el periodo comprendido entre 1992 y 2014 el valor agregado en términos reales de los "otros servicios prestados a empresas" crecieron a una tasa promedio de 9,2 \%, cifra superior inclusive a la tasa de crecimiento promedio del grupo de actividades orientadas a los servicios $(5,1 \%)$ y a la de actividades que han sido tradicionales como la agricultura, silvicultura y pesca $(3,2 \%)$ y la manufactura $(5 \%)$.

Desde el punto de vista de la economía espacial, la transformación del sistema productivo y la mayor inserción en la economía mundial ha propiciado un mayor encadenamiento entre actividades y productos; con ello un mayor dinamismo de la economía y el empleo en las fases expansivas del ciclo económico, no obstante, Arias y Sánchez (2009) destacan que este 
dinamismo se tiende a concentrar principalmente en la región central ${ }^{2}$ del país. Las asimetrías y fricciones propias de otras regiones de planificación han impedido un mayor efecto derrame, el cual provoca en muchos casos desigualdades en el crecimiento, el empleo y las remuneraciones.

A manera de ejemplo se puede citar el caso de las regiones que focalizan importantes actividades asociadas con servicios y la producción de bienes que se venden en el exterior, como es el caso de la región Chorotega y Pacífico Central, que no han podido aprovechar su dinamismo y tener efectos positivos de reactivación económica y del empleo a nivel local (Arias y Sánchez, 2009, op.cit) debido a que tiende a importarse mano de obra calificada de otras regiones a estas zonas, dado que la oferta laboral local no cumple con los estándares adecuados para ser parte del proceso productivo.

El objetivo del presente trabajo es examinar y analizar los encadenamientos productivos a partir de la información contenida en la MIP-2011 de la economía total por producto, elaborada por el Banco Central de Costa Rica. Esto con el fin de brindar un elemento técnico para la discusión de las acciones que pueden implementarse para acelerar el crecimiento de la economía y el empleo a partir de la identificación de las actividades económicas que tienen un mayor efecto dentro de la estructura productiva.

Asimismo, en la literatura sobre el tema, es reconocido que el estudio de los encadenamientos permite la formulación de escenarios probables y deseables que sirvan para definir adecuadamente metas de crecimiento y desarrollo económico. Adicionalmente, es posible identificar si la ausencia de encadenamientos productivos y la falta de estímulos a actividades con ventajas relativas acentúan los problemas del crecimiento y el desempleo.

Para el Banco Central de Costa Rica, la MIP es una fuente de información valiosa a partir de la cual se puede desarrollar investigación económica que apoye a las autoridades en el logro del objetivo estratégico de la institución 3 "Profundizar en el análisis de la capacidad de crecimiento potencial de la economía, a fin de medir el impacto de las políticas macroeconómicas del Banco Central y de las demás políticas públicas".

El documento se estructura de la siguiente forma: en la sección dos se analiza brevemente la evolución de la composición del producto interno bruto (PIB) por actividades productivas. La tercera y cuarta sección contiene una reseña de los principales temas metodológicos en torno a

\footnotetext{
2 Las regiones socioeconómicas de Costa Rica son una subdivisión político-económica realizada por Decreto Ejecutivo № 7944 del 26 de enero de 1978. Estas regiones son seis en total: Región Central, Región Chorotega, Región Pacífico Central, Región Brunca, Región Huetar Atlántica y Región Huetar Norte.

${ }^{3}$ De acuerdo con el Plan Estratégico del BCCR 2015-2018 de Banco Central de Costa Rica.
}

4 
la construcción de la MIP-2011 y al cálculo de los encadenamientos productivos. Seguidamente en la quinta sección se comentan los principales resultados. Finalmente, se presentan las principales conclusiones y recomendaciones.

\section{Evolución reciente de las actividades productivas en Costa Rica}

Como se mencionó en párrafos previos con información disponible para el período 1992 y 2014 se constata que la economía costarricense continúa experimentando un cambio en su estructura productiva ${ }^{4}$. Dicho cambio ha supuesto al igual que en otras economías, una mayor participación como porcentaje del PIB de las actividades que se orientan a la producción de servicios.

Tal y como se puede apreciar en la figura 1 la importancia relativa de dicho sector se ha incrementado en casi 12 p.p entre los años previamente mencionados, este proceso de cambio se da en detrimento de las participaciones de las actividades pertenecientes al sector primario y secundario. Lo anterior es mucho más evidente cuando se analizan cuáles son las actividades económicas dentro de dichas agrupaciones que presentan un mayor crecimiento y dinamismo.

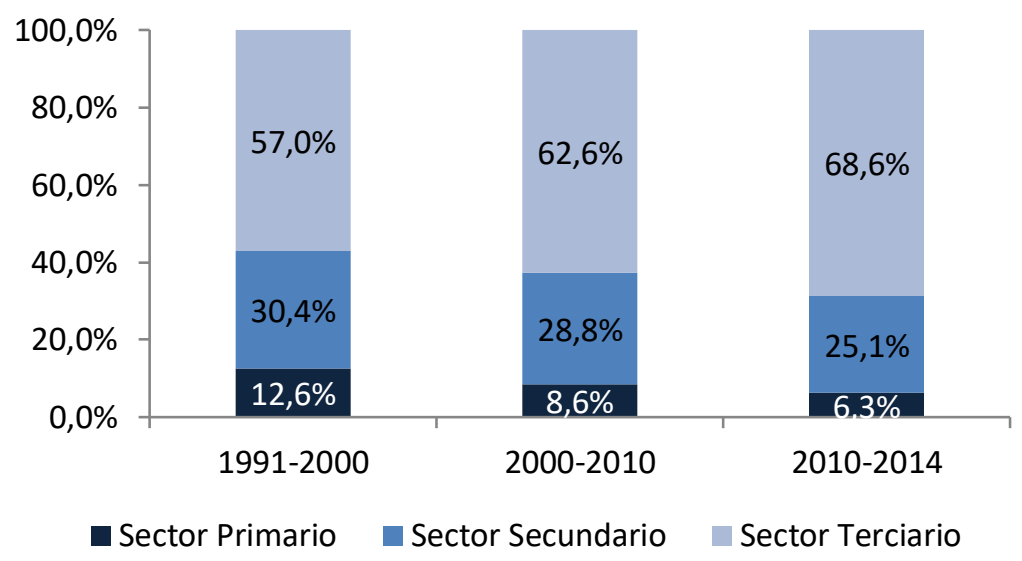

Figura 1. Estructura de la producción según sector productivo sobre la base de cifras nominales. Fuente: elaboración propia con base en las cifras del Departamento de Estadística Macroeconómica del Banco Central de Costa Rica (BCCR).

\footnotetext{
${ }^{4}$ Es relevante mencionar que el cambio en la estructura productiva se refiere a una situación en la cual la participación de las actividades cambia en el tiempo, esto quizás es un proceso dinámico que se experimenta desde los años 80's sin embargo por no disponer de datos desagregados de las cuentas nacionales para ese periodo la referencia para el análisis se inicia a partir del año 1992. Esta aseveración no constituye una hipótesis de cambio estructural, ya que no se puede corroborar dado la ausencia de datos en el marco de una matriz de insumo producto.
} 
Seguidamente, la tabla 1 resume la información relativa a las tasas de crecimiento de las industrias que se agrupan dentro de los sectores primario, secundario y terciario; destacan dentro del sector terciario los "otros servicios prestados a empresas" 5 , los servicios de "transporte, almacenaje y comunicaciones, los "establecimientos financieros y seguros" y las actividades relacionadas con el turismo como el "comercio, restaurantes y hoteles".

Tabla 1.

Tasas de crecimiento promedio del valor agregado de las industrias pertenecientes al sector primario, secundario y terciario.

\begin{tabular}{|c|c|c|c|}
\hline & $2000-2010$ & $2010-2014$ & 1992-2014 \\
\hline Sector Primario & $2,7 \%$ & $3,1 \%$ & $3,2 \%$ \\
\hline Agricultura, silvicultura y pesca & $2,7 \%$ & $3,2 \%$ & $3,2 \%$ \\
\hline Explotación de minas y canteras & $2,4 \%$ & $0,0 \%$ & $2,4 \%$ \\
\hline Sector Secundario & $3,2 \%$ & $3,4 \%$ & $4,9 \%$ \\
\hline Industria manufacturera & $2,6 \%$ & $4,0 \%$ & $5,0 \%$ \\
\hline Construcción & $6,8 \%$ & $1,0 \%$ & $5,6 \%$ \\
\hline Electricidad y agua & $4,1 \%$ & $2,2 \%$ & $4,2 \%$ \\
\hline Sector Terciario & $5,1 \%$ & $5,0 \%$ & $5,1 \%$ \\
\hline Comercio, restaurantes y hoteles & $2,7 \%$ & $3,9 \%$ & $4,0 \%$ \\
\hline Transporte, almacenaje y comunicaciones & $9,5 \%$ & $6,4 \%$ & $8,6 \%$ \\
\hline Establecimientos financieros y seguros & $8,0 \%$ & $5,6 \%$ & $6,8 \%$ \\
\hline Bienes Inmuebles & $3,2 \%$ & $3,6 \%$ & $3,2 \%$ \\
\hline Otros servicios prestados a empresas & $11,9 \%$ & $9,6 \%$ & $9,2 \%$ \\
\hline Servicios de administración pública & $2,5 \%$ & $1,8 \%$ & $1,7 \%$ \\
\hline Servicios comunales, sociales y personales & $3,3 \%$ & $3,3 \%$ & $3,5 \%$ \\
\hline $\begin{array}{l}\text { Menos: servicios de intermediación financiera } \\
\text { medidos indirectamente (SIFMI) }\end{array}$ & $9,3 \%$ & $7,0 \%$ & $9,1 \%$ \\
\hline
\end{tabular}

Fuente: elaboración propia con base en las cifras del Departamento de Estadística Macroeconómica (DEM) del BCCR.

Para el mismo período de referencia y como complemento a dicha evolución, cabe mencionar que las exportaciones de servicios crecieron a una tasa promedio de 7,63\%, cifra superior en 1,34 p.p. al crecimiento promedio experimentado por las exportaciones de bienes.

Adicionalmente, el comportamiento de las industrias que conforman el conjunto de actividades orientadas a los servicios se ha fortalecido en los años recientes por la creciente tendencia en la apertura de centros de servicios compartidos y de operaciones por parte de diferentes

${ }^{5}$ La lista de actividades que forman parte de esta definición de actividades se encuentra en el anexo 1.

6 
empresas tanto nacionales como multinacionales. De acuerdo con información disponible en la base de datos "Directorio de Empresas del BCCR" se logró identificar por ejemplo, que entre el año 2006 y 2013 el número promedio ${ }^{6}$ de empresas nuevas que inician operaciones productivas relacionadas con los servicios es de 3.871 por año, en comparación con la manufactura cuyo número medio de empresas nuevas es de 98 (figura 2).

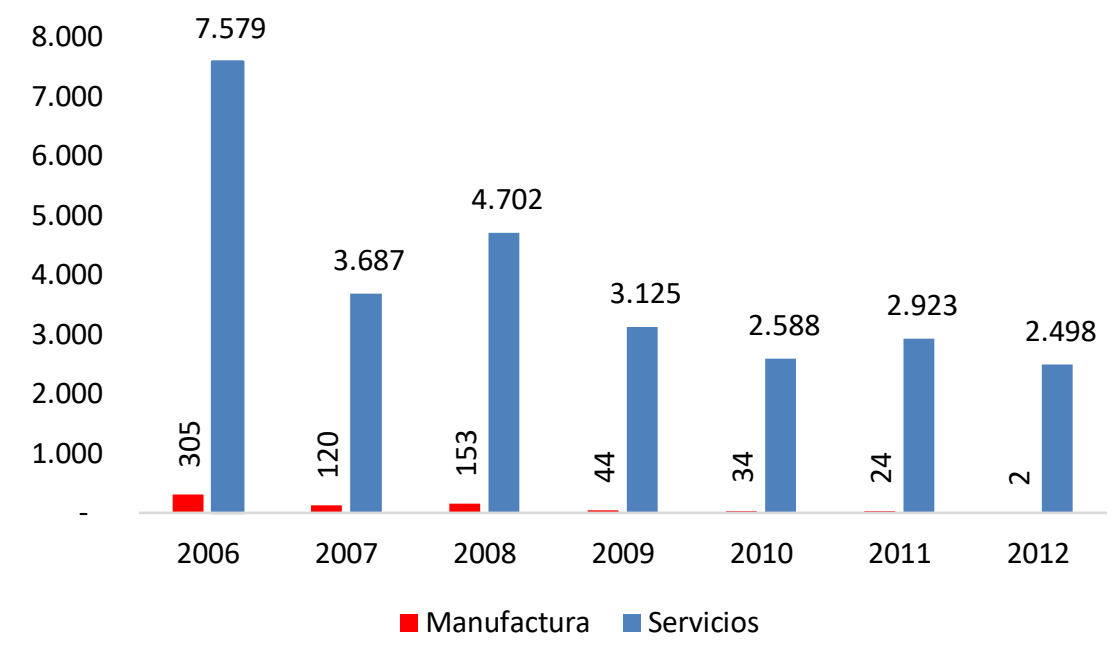

Figura 2. Empresas nuevas relacionadas con servicios y la manufactura, BCCR. Fuente: elaboración propia.

Ahora bien, utilizando la misma fuente de información, se logra identificar que del total de empresas ${ }^{7}$ vigentes al año 2011 cerca del 91,57\% corresponde a empresas del sector servicios y comercio.

Otro tema importante a la hora de analizar los encadenamientos productivos tiene que ver con el empleo y los salarios. En relación con lo primero y tomando como referencia el número de trabajadores reportados a la seguridad social se observa que el sector terciario engloba más del 80 \% del empleo formal reportado y que a partir de 2008 crea más empleo en comparación con el sector primario y secundario. A manera de ejemplo, en la época poscrisis (2009-2012) dicho sector reportó de forma acumulada la creación de 91.076 nuevos empleos formales (tabla 2); estos puestos se distribuyen principalmente en actividades como enseñanza, comercio al por mayor y actividades administrativas y de apoyo.

\footnotetext{
${ }^{6}$ Este resultado se obtiene como la diferencia de las nuevas empresas que aparecen por primera vez con registros administrativos menos las empresas que mantenían registros contables en el año previo. Es importante mencionar que a pesar de ser empresas con cédula jurídica, su tamaño según el empleo puede variar, esto quiere decir que dentro de esta estimación se toman pequeñas, medianas y grandes empresas.

${ }^{7}$ Con cédulas jurídicas y físicas con más de un empleado.
} 
En relación con el grado de especialización del personal ocupado, según la información de la MIP-2011 el 92,61\% del personal ocupado para la elaboración de productos del sector primario es "no calificado"; para el caso de los productos elaborados por la manufactura (sector secundario) la mayor proporción de ocupados se cataloga como "calificación media" (52,02 \%) y finalmente el sector terciario ocupa un $36 \%$ de personal calificado alto y un $45 \%$ de ocupados con "calificación media".

Tabla 2.

Trabajadores reportados a la seguridad social promedio anual y variación absoluta

\begin{tabular}{lccccccc}
\hline Sector/año & $\mathbf{2 0 0 6}$ & $\mathbf{2 0 0 7}$ & $\mathbf{2 0 0 8}$ & $\mathbf{2 0 0 9}$ & $\mathbf{2 0 1 0}$ & $\mathbf{2 0 1 1}$ & $\mathbf{2 0 1 2}$ \\
\hline Primario & 84,755 & 87,932 & 91,078 & 88,690 & 88,390 & 82,254 & 83,976 \\
Var. Absoluta & $\mathbf{3 , 5 1 1}$ & $\mathbf{3 , 1 7 7}$ & $\mathbf{3 , 1 4 6}$ & $\mathbf{- 2 , 3 8 8}$ & $-\mathbf{3 0 0}$ & $-\mathbf{6 , 1 3 6}$ & $\mathbf{1 , 7 2 2}$ \\
Secundario & 83,882 & 87,504 & 87,476 & 78,436 & 80,411 & 83,410 & 85,419 \\
Var. Absoluta & $\mathbf{5 , 2 4 9}$ & $\mathbf{3 , 6 2 2}$ & $-\mathbf{2 8}$ & $-\mathbf{9 , 0 4 0}$ & $\mathbf{1 , 9 7 5}$ & $\mathbf{2 , 9 9 9}$ & $\mathbf{2 , 0 0 9}$ \\
Terciario & $\mathbf{7 4 3 , 3 3 6}$ & 805,285 & 869,004 & 879,311 & 892,164 & 929,540 & 960,081 \\
Var. Absoluta & $\mathbf{6 4 , 0 4 1}$ & $\mathbf{6 1 , 9 4 9}$ & $\mathbf{6 3 , 7 1 8}$ & $\mathbf{1 0 , 3 0 8}$ & $\mathbf{1 2 , 8 5 3}$ & $\mathbf{3 7 , 3 7 6}$ & $\mathbf{3 0 , 5 4 1}$ \\
\hline Total & $\mathbf{9 1 1 , 9 7 3}$ & $\mathbf{9 8 0 , 7 2 1}$ & $\mathbf{1 , 0 4 7 , 5 5 8}$ & $\mathbf{1 , 0 4 6 , 4 3 7}$ & $\mathbf{1 , 0 6 0 , 9 6 5}$ & $\mathbf{1 , 0 9 5 , 2 0 4}$ & $\mathbf{1 , 1 2 9 , 4 7 6}$ \\
\hline
\end{tabular}

Fuente: elaboración propia.

Finalmente, y en relación con los ingresos, el salario promedio mensual en términos reales ${ }^{8}$ para el caso de actividades como por ejemplo comercio y las actividades de servicios es de \$276.085 y $\$ 278.938$ respectivamente. Estos montos son levemente superiores a los reportados en el sector agrícola y manufacturero (\$214.754 y $\$ 270.706$, respectivamente).

Todo lo anterior constituye un marco de referencia para analizar la estructura productiva a la luz de la información que provee la MIP-2011, especialmente, para identificar las estructuras productivas más relevantes desde la perspectiva de los encadenamientos productivos.

\section{Construcción de la MIP para Costa Rica}

Una matriz de insumo producto es una representación en forma matricial del equilibrio entre oferta y utilización que se da en la economía. La matriz de insumo producto puede elaborarse tanto para un país, como para una región dado que permite analizar y medir la producción que

${ }^{8}$ Colones constantes de 2015

8 
satisfacen ciertos niveles de consumo e inversión; como constituye una descripción sintética de la economía, las estructuras que se estiman a lo interno de la MIP suelen utilizarse también para generar proyecciones de las necesidades de producción ante cambios en la demanda.

En Costa Rica existen estudios que abordaron el tema de la construcción de una matriz de insumo producto para la economía costarricense, entre ellas la elaborada por Leiva y Vargas (1972), Bulmer-Thomas (1976), el Instituto de Investigaciones Económicas de la Universidad de Costa Rica en 1985, Sánchez (2006) para el año 2012 en el marco del proyecto MACEPES ${ }^{9}$ y más recientemente el Banco Central de Costa Rica para el año 2011 (MIP-2011) y su versión 2012.

Una de las principales motivaciones que propició el desarrollo de la MIP-2011 para Costa Rica es la creciente importancia del estudio de las cadenas globales de valor en la economía (Gereffi y Sturgeon, 2005$)^{10}$. Con el fin de medir el valor agregado que genera el comercio internacional en la economía local, varias instituciones suscribieron un convenio de cooperación con el Banco Central de Costa Rica para proceder con la construcción de esta herramienta ${ }^{11}$. Las recomendaciones internacionales, entre ellas las consignadas en el Sistema de Cuentas Nacionales 2008, ( $\underline{\text { SCN 2008) }}$ indican que para elaborar una matriz insumo producto, primero se debe disponer de un cuadro de oferta y utilización (COU).

En línea con lo anterior el Departamento de Estadística Macroeconómica (DEM) elaboró un nuevo cuadro de oferta y utilización (COU) base 2011, dado que la estructura del que estaba disponible tiene como base 1991. El nuevo COU cuenta con 136 actividades económicas (CIIU4) y 183 productos (a nivel de la CCP2.0).

A partir de los cuadros de oferta y utilización, existen dos posibilidades para obtener una matriz insumo producto: la primera es expresar la matriz sólo en términos de productos términos de industrias y la segunda en términos de productos. La matriz construida y utilizada para este trabajo consta de una dimensión de 76x76 productos. Las principales fuentes de información para la MIP-2011 de Costa Rica se consignan en la tabla 3.

\footnotetext{
${ }^{9}$ PNUND-Modelado macro-micro de equilibrio general para el análisis del impacto de políticas macroeconómicas y otros choques sobre el crecimiento, los balances macroeconómicos, la desigualdad y la pobreza.

10 Para mayor detalle Humphrey y Schimtz (2002). "How does insertion in global value chains affect upgrading in industrial clusters?"

11 Las instituciones participantes fueron la Promotora de Comercio Exterior (PROCOMER), el Ministerio de Comercio Exterior (COMEX) y el Banco Central de Costa Rica (BCCR) en el contexto del Proyecto de Cambio de Año de Referencia de las Cuentas Nacionales.
} 
Tabla 3.

Fuentes de información de la MIP-2011

\begin{tabular}{|c|c|}
\hline Vector de producción & $\begin{array}{l}\text { Registro de Variables Económicas de Empresas y Establecimientos del } \\
\text { BCCR ( } 26500 \text { empresas). }\end{array}$ \\
\hline $\begin{array}{l}\text { Vector de consumo final de } \\
\text { hogares }\end{array}$ & $\begin{array}{l}\text { Encuesta de Ingresos y Gastos de los Hogares de } 2004 \text { (ENIGH) } \\
\text { extrapolada al } 2011 \text { y registros administrativos de ventas del Ministerio de } \\
\text { Hacienda. }\end{array}$ \\
\hline Vector de formación de capital & $\begin{array}{l}\text { Principalmente registros de comercio exterior y estimaciones de la } \\
\text { actividad de la construcción. }\end{array}$ \\
\hline $\begin{array}{l}\text { Vector de exportaciones e } \\
\text { importaciones }\end{array}$ & Registros de comercio exterior y de balanza de pagos. \\
\hline Impuestos a los productos & $\begin{array}{l}\text { IVA y selectivo de consumo: con base en la normativa legal se determinó } \\
\text { la lista de productos que están sujetos de este gravamen. Se utilizó el } \\
\text { vector de consumo final y el consumo intermedio de las actividades } \\
\text { productivas como referencia para distribuir el dato de recaudación. } \\
\text { Impuestos a la importación: distribución por producto con base a los } \\
\text { registros de la Dirección General de Aduanas }\end{array}$ \\
\hline Importaciones & $\begin{array}{l}\text { Registros de comercio exterior. Para importadores comerciantes se utiliza } \\
\text { la información de la declaración D151 del Ministerio de Hacienda para } \\
\text { aproximar el destino de sus ventas en consumo final, consumo intermedio } \\
\text { o formación de capital. }\end{array}$ \\
\hline $\begin{array}{l}\text { Matriz de márgenes de comercio y } \\
\text { transporte }\end{array}$ & $\begin{array}{l}\text { Se calcularon coeficientes de márgenes de comercio para cada producto } \\
\text { según las diferentes utilizaciones: consumo intermedio, consumo final, } \\
\text { formación de capital y exportaciones. }\end{array}$ \\
\hline
\end{tabular}

Fuente: elaboración propia con información proporcionada por el Departamento de Estadística Macroeconómica del BCCR.

En lo que respecta a las estructuras de costos se utilizó información del nuevo año de referencia de las Cuentas Macroeconómicas (2012), según las diferentes industrias: agrícola, manufacturera, comercial, de servicios, servicios de administración pública, financieros, etc. Por ejemplo, para el caso de la industria agrícola se utiliza información de 100 estudios de caso para 38 productos. Para la manufactura, comercio y servicios se utiliza información de 450 empresas. En el caso de la construcción se usan registros administrativos del Colegio Federado de Ingenieros y Arquitectos (CFIA), empresas públicas y el gobierno. Todo el proceso de compilación de la MIP-2011 se basa en la adopción del Sistema de Cuentas Nacionales (en adelante SCN) del 2008, el cual introduce una serie de recomendaciones que difieren de lo que establece el SCN 1993.

\section{Metodología para el cálculo de los encadenamientos productivos}

En el análisis de encadenamientos se pueden describir dos tipos: hacia atrás y hacia adelante. Los primeros miden la capacidad de un sector para impulsar directamente a otros relacionados 10 
con este por la demanda de bienes de consumo intermedio, luego de que un choque exógeno estimula la actividad de tales sectores. Los segundos miden la capacidad de un sector para estimular a otros, por su capacidad de oferta u otra forma de servir como insumo dentro de otros sectores. Ambos evalúan la magnitud de las transacciones entre industrias.

El presente estudio utiliza la información por producto de la MIP del año 2011. Se eliminan aquellos productos que no tienen registros tanto en las columnas como en las filas ${ }^{12}$. Con esto la dimensión de la matriz original de 76x76 se reduce a una de 60x60 productos.

Los coeficientes técnicos se estiman siguiendo la metodología que se cita en Miller y Blair (2009), quien a su vez toma como base los trabajos pioneros de Chenery y Watanabe (1958),(op.cit), Hirschman(op.cit) y Rasmussen (1958) y que cuenta con aplicaciones recientes en Claus(2003), Kula (2008), Robles y Polanco (2011) y Hernández (2012).

Bajo el supuesto de tecnología constante ${ }^{13}$ tanto en la producción de cada producto, así como en el consumo de cada bien se parte de lo siguiente:

$$
\text { (1.1) } X=A X+Y
$$

La expresión (1.1) constituye un sistema de $n$ ecuaciones con $n$ incógnitas. $X$ es un vector de $n \times 1$ que representa los productos que se producen en la economía y cada uno de los componentes $X_{i}$ es la producción del producto $i$. Adicionalmente, $Y$ es un vector $n x m$ donde cada columna es cada uno de los componentes de la demanda final. A es la matriz de coeficientes técnicos ( $\left.a_{i j}\right)$, estos a su vez se definen de la siguiente forma:

$$
\text { (1.2) } a_{i j}=\frac{x_{i j}}{x_{j}}
$$

Donde $X_{i j}$ es la producción de cada producto y $X_{j}$ la producción total de lo $j$ productores. Al resolver la ecuación (1.1) para $X$, se obtiene que:

$$
\text { (1.3) } X=(I-A)^{-1} Y=B^{*} Y
$$

De lo anterior se desprende que $B$ es una matriz que representa los requerimientos totales de la economía. Los elementos de la matriz $A$ son las cantidades de insumos que se requieren para

\footnotetext{
${ }^{12}$ Ejemplos: petróleo crudo y gas natural, hilados, construcción y reparación de barcos. La matriz de $76 \times 76$ fue elaborada para mantener la homogeneidad de la estructura de la MIP para poder ser integrada a la MIP utilizada para el estudio de las cadenas globales de valor.

${ }^{13}$ Todos los sectores utilizan la misma tecnología y se tienen los mismos niveles de eficiencia.
} 
producir una unidad de producto. Por su parte la matriz (I-A) ${ }^{-1}$ muestra el impacto total o efecto multiplicador de un incremento exógeno de la demanda final. Por ejemplo: un incremento de una unidad en la producción de arroz lleva a la interacción y al movimiento de una cadena de producción que involucra productos como fertilizantes y agroquímicos, electricidad y gas, abastecimiento de agua, transporte, entre otros servicios.

Miller y Blair (2009) (op.cit) citan que Chenery y Watanabe proponen el cálculo de los indicadores hacia atrás y hacia adelante con base en los coeficientes técnicos ${ }^{14}$.

Los primeros se calculan de la siguiente forma:

$$
\text { (1.4) } D B L_{j}=\frac{\sum_{i=1}^{n} x_{i j}}{X_{j}}=\sum_{i} a_{i j}
$$

Y los encadenamientos directos hacia delante de la siguiente forma:

$$
\text { (1.5) } D F L_{i}=\frac{\sum_{j=1}^{n} x_{i j}}{X_{i}}=\sum_{j} a_{i j}
$$

Adicionalmente, una vez obtenidos los valores de los encadenamientos se procede a agrupar los de cada producto de acuerdo con la siguiente clasificación sectorial:

\section{Tabla 4.}

\section{Clasificación sectorial}

$\begin{array}{cc}\text { Condición } & \text { Clasificación } \\ D B L_{j}<\frac{\sum_{j=1}^{n} D B L_{j}}{n} \text { y } D F L_{i}<\frac{\sum_{i=1}^{n} D F L_{i}}{n} & \text { No manufactureras/Destino Final } \\ D B L_{j} \geq \frac{\sum_{j=1}^{n} D B L_{j}}{n} \text { y } D F L_{i}<\frac{\sum_{i=1}^{n} D F L_{i}}{n} & \text { Manufactureras/Destino Final }\end{array}$

${ }^{14} \mathrm{DBL}=$ Encadenamiento directo hacia atrás y DFL= Encadenamiento directo hacia adelante.

12 


\begin{tabular}{cc}
\hline Condición & Clasificación \\
$D B L_{j}<\frac{\sum_{j=1}^{n} D B L_{j}}{n}$ y $D F L_{i} \geq \frac{\sum_{i=1}^{n} D F L_{j}}{n}$ & No manufactureras/Destino Intermedio \\
$D B L_{j} \geq \frac{\sum_{j=1}^{n} D B L_{j}}{n}$ y $D F L_{i} \geq \frac{\sum_{i=1}^{n} D F L_{j}}{n}$ & Manufactureras/Destino intermedio \\
\hline
\end{tabular}

Fuente: elaboración propia con base en Schuschny (2005).

Donde los productos clasificados como "No manufactureras/Destino Final" corresponden a productos con bajo encadenamiento hacia adelante y hacia atrás cuya producción se destina principalmente a satisfacer la demanda final. La clasificación "Manufactureras/Destino Final" son aquellos productos que presentan un bajo encadenamiento hacia adelante y alto hacia atrás y pueden afectar en mayor cuantía al crecimiento de la economía, por la posibilidad que tienen de inducir otras actividades.

Por otro lado, se tiene las "No manufactureras/Destino Intermedio" y las "Manufactureras/Destino Intermedio". El primer grupo corresponde a aquellos productos que tienen un alto encadenamiento directo hacia adelante y bajo hacia atrás y cuya producción es básicamente utilizada como consumo intermedio; el segundo grupo se caracteriza por registrar altos encadenamientos hacia adelante y hacia atrás, son productos cuya producción requiere una alta cantidad de insumos para luego vender su producción para la elaboración de otros productos.

\section{Principales resultados}

Siguiendo la metodología descrita en la sección anterior para cuantificar los encadenamientos productivos se ha logrado identificar que los productos con mayores encadenamientos hacia atrás son aquellos que están relacionados con actividades manufactureras como es el caso de los "productos cárnicos y productos lácteos", la "madera", los "otros productos alimenticios", "granos y harina molida", entre otros. Esto supone que cada vez que la demanda de estos productos se incremente, la producción de los insumos necesarios satisface dicha demanda también.

Un elemento característico es que por lo general los productos asociados con servicios suelen tener bajos encadenamientos hacia atrás. Esto es el caso de los "otros servicios y el "comercio al por mayor y al detalle". 
En el caso de los encadenamientos hacia adelante y a diferencia de lo expresado en el párrafo anterior, los "otros servicios", el "comercio al por mayor y al detalle" y las "finanzas y seguros" son los productos más importantes según los encadenamientos. Estos afectan a más productos, es decir, inciden en la estructura de costos de la producción de otros productos desde un punto de vista de oferta. La tabla 5 contiene un resumen con los 20 productos más importantes según cada tipo de encadenamiento.

Tabla 5.

Encadenamientos directos hacia atrás y hacia adelante

\begin{tabular}{|c|c|c|c|c|}
\hline & Producto & $\begin{array}{l}\text { Encadenamiento } \\
\text { hacia atrás }\end{array}$ & Producto & $\begin{array}{c}\text { Encadenamiento } \\
\text { hacia adelante }\end{array}$ \\
\hline 1 & $\begin{array}{l}\text { Productos cárnicos y productos } \\
\text { lácteos }\end{array}$ & 0,73 & Otros servicios & 3,48 \\
\hline 2 & Madera & 0,60 & $\begin{array}{c}\text { Comercio al por mayor y } \\
\text { al detalle }\end{array}$ & 2,51 \\
\hline 3 & Otros productos alimenticios & 0,55 & Finanzas y seguros & 2,07 \\
\hline 4 & Granos y harina molida & 0,54 & Electricidad y gas & 0,88 \\
\hline 5 & Productos pesqueros & 0,50 & Transporte & 0,83 \\
\hline 6 & Otras construcciones & 0,50 & Construcción de edificios & 0,63 \\
\hline 7 & Hoteles & 0,49 & $\begin{array}{l}\text { Otros productos } \\
\text { alimenticios }\end{array}$ & 0,57 \\
\hline 8 & Vidrio y productos de vidrio & 0,48 & Ganado y aves de corral & 0,57 \\
\hline 9 & Tabaco & 0,45 & Bienes raíces & 0,57 \\
\hline 10 & $\begin{array}{l}\text { Cemento y productos de } \\
\text { cemento }\end{array}$ & 0,45 & $\begin{array}{c}\text { Fertilizantes y pesticidas } \\
\text { químicos }\end{array}$ & 0,56 \\
\hline 11 & Otros equipos de transporte & 0,44 & Papel y pulpa de papel & 0,50 \\
\hline 12 & Arroz & 0,43 & Arroz & 0,48 \\
\hline 13 & Restaurantes & 0,42 & Silvicultura & 0,44 \\
\hline 14 & $\begin{array}{l}\text { Minerales no metálicos y } \\
\text { canteras }\end{array}$ & 0,41 & $\begin{array}{c}\text { Teléfono y } \\
\text { telecomunicaciones }\end{array}$ & 0,43 \\
\hline 15 & Impresión y publicación & 0,40 & $\begin{array}{l}\text { Refinado de petróleo y sus } \\
\text { productos }\end{array}$ & 0,37 \\
\hline 16 & Otros productos químicos & 0,38 & Pesca & 0,33 \\
\hline 17 & Papel y pulpa de papel & 0,37 & Cultivos alimentarios & 0,32 \\
\hline 18 & Pesca & 0,36 & Productos de plástico & 0,31 \\
\hline 19 & Otros productos manufactureros & 0,36 & $\begin{array}{l}\text { Accesorios de iluminación, } \\
\text { baterías, cableado y otros }\end{array}$ & 0,29 \\
\hline 20 & $\begin{array}{l}\text { Televisores, radios, audios y } \\
\text { equipos de comunicación }\end{array}$ & 0,36 & Productos metálicos & 0,26 \\
\hline
\end{tabular}

14 
El resultado anterior constituye uno de los elementos más característicos del análisis de insumo producto en el contexto de la toma de decisiones y el diseño de políticas públicas orientadas al estímulo productivo, ya que permite identificar cuáles son los productos más importantes de acuerdo a los encadenamientos estimados, como se puede apreciar la base productiva del país se concentra en actividades como la manufactura y los servicios que son los mayores generadores de empleo en el país.

Con el fin de separarse del análisis descriptivo tradicional, el presente trabajo toma en consideración otros indicadores como referencia para establecer la importancia de los productos.

\section{Análisis de encadenamientos hacia atrás según importancia relativa}

En primer lugar, se lleva a cabo un ordenamiento de los productos, en donde se toma como referencia la participación dentro de la demanda final, segundo, la importancia dentro de las exportaciones y finalmente la participación según el personal ocupado para ambos tipos de encadenamiento. Lo anterior pretende servir de referencia para una mejor y más clara identificación de los productos con alto potencial para incrementar el crecimiento económico y dinamizar el empleo.

Por ejemplo, los 15 productos más importantes según la demanda final representan alrededor de $78,6 \%$ de participación relativa y además tienen un alto número de vínculos con otros productos. Entre ellos destacan "otros servicios", "comercio al por mayor y al detalle", "educación e investigación", "construcción de edificios", "bienes raíces" y "otros productos alimenticios", estos tienen la característica de tener bajos encadenamientos hacia atrás, es por ello que una política diseñada para acelerar el crecimiento y el empleo basada en un criterio de importancia en la demanda final podría no provocar los efectos esperados debido a su poca capacidad de influir sobre otros desde la perspectiva de la demanda. Caso contrario sucede con productos con menor participación relativa, pero con mayores encadenamientos hacia atrás como los "productos cárnicos y productos lácteos", "madera", "otros productos alimenticios", "granos y harina molida" y "productos pesqueros". 


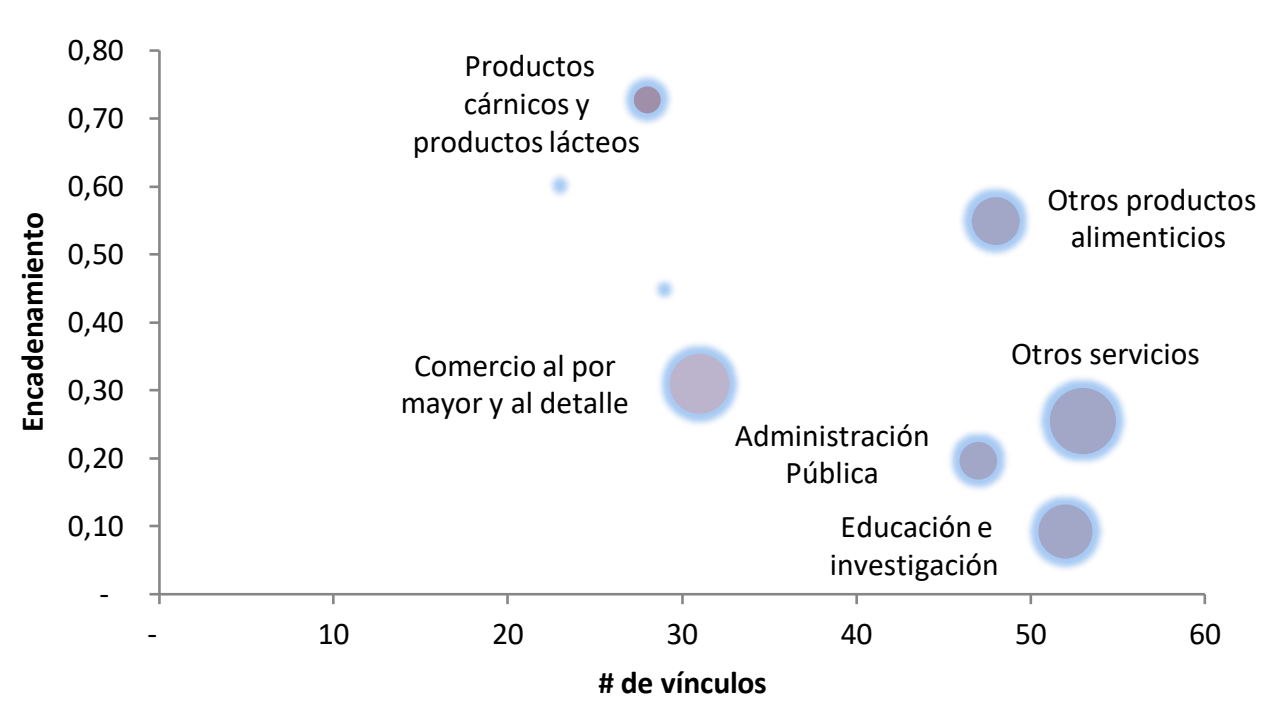

Figura 3. Encadenamientos hacia atrás e importancia relativa según demanda final. Nota: el ancho de la burbuja mide la importancia relativa dentro de la demanda final, las exportaciones o el personal ocupado. Fuente: elaboración propia.

Un criterio adicional puede ser el caso de las exportaciones (figura 3), para este caso la importancia relativa de cada producto viene dada por su participación dentro del total de exportaciones, bajo este criterio nuevamente los productos relacionados con los servicios son los más importantes, los "otros servicios", "otros productos alimenticios", "cultivos alimentarios" y "máquinas de precisión", estos representan cerca del 52\% de las exportaciones. No obstante, estos productos no son capaces de generar altos encadenamientos hacia atrás como el caso de los "productos cárnicos y productos lácteos", "madera", "otros productos alimenticios", "granos y harina molida", "productos pesqueros", "otras construcciones", "hoteles y el vidrio y productos de vidrio".

16 


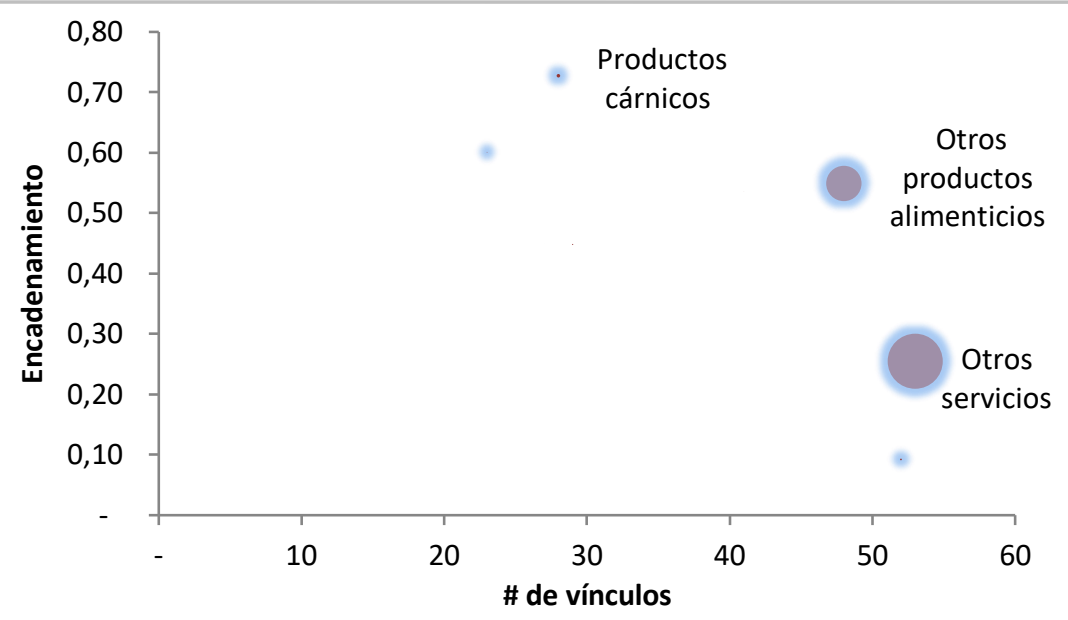

Figura 4. Encadenamientos hacia atrás e importancia relativa según exportaciones. Fuente: elaboración propia.

Finalmente, los productos que tienen mayor porcentaje de participación en personal ocupado son los productos que están relacionadas con los servicios y no son capaces de producir mayores cambios en el número de ocupados ante aumentos en la demanda, dado su bajo nivel de encadenamiento hacia atrás.

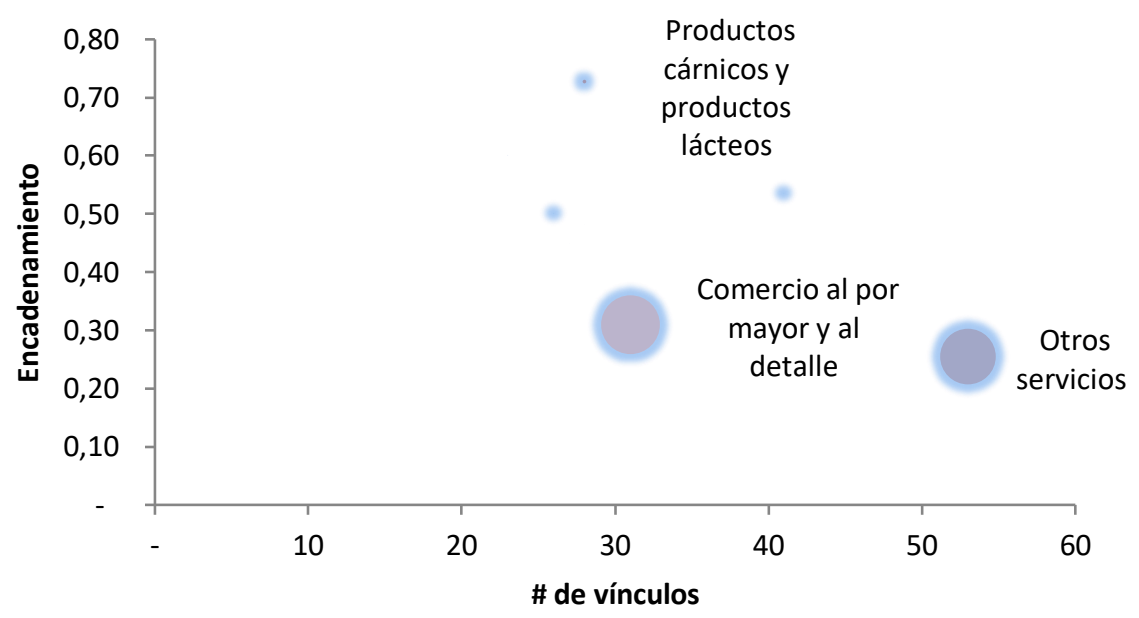

Figura 5. Encadenamientos hacia atrás e importancia relativa según personal ocupado. Fuente: elaboración propia. 


\section{Análisis de encadenamientos hacia adelante según importancia relativa}

Esta sección contiene una descripción similar al del punto anterior, pero basada en los encadenamientos hacia adelante.

Como se puede apreciar en la figura 7, los productos con mayores encadenamientos son los que a su vez tienen los mayores encadenamientos con otros productos y adicionalmente son los más importantes dentro de la demanda final, las exportaciones y el personal ocupado. Este el caso de los "otros servicios" y el "comercio al por mayor y al detalle" principalmente. Es decir estos productos independientemente del criterio de referencia que se utilice, tienen la capacidad de estimular a otras actividades al satisfacer el consumo intermedio de estos.
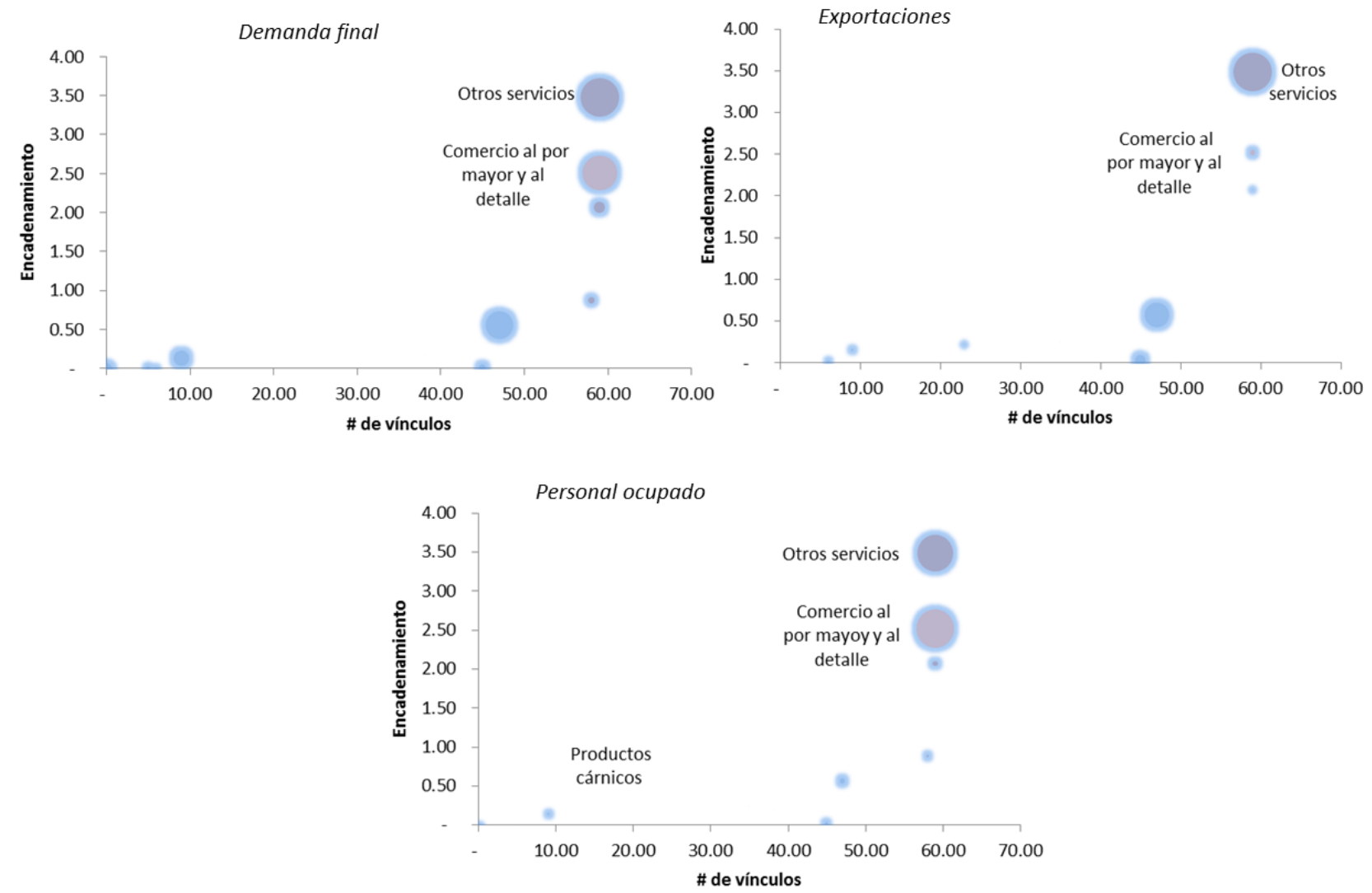

Figura 7. Encadenamientos hacia adelante e importancia relativa. Fuente: elaboración propia.

\section{Resultados de la clasificación sectorial}

Con la información anterior también se lleva a cabo una clasificación sectorial. De los 60 productos de los cuales se tiene información en la MIP, ocho se clasifican como 18 
"Manufactureras/Destino Intermedio" es decir, son productos que demandan y ofrecen grandes cantidades de insumos intermedios y por lo tanto son paso obligado de los flujos de la economía. Tal es el caso de: "arroz", "pesca", "construcción de edificios", "teléfono y telecomunicaciones", "finanzas y seguros", entre otros. Por otra parte, 19 actividades se clasifican como "Manufactureras/Destino Final": son grandes demandantes de insumos intermedios, pueden afectar en mayor cuantía al crecimiento de la economía por la posibilidad que tienen de inducir en la producción de otros productos. Este es el caso de: "otras construcciones", "restaurantes", "hoteles", "otros productos cárnicos y lácteos", "medicamentos", "otros productos manufactureros", entre otros.

Adicionalmente, 24 productos se clasifican como "No manufactureras/Destino Final", este es el caso de productos que consumen una cantidad baja de insumos y su producción se destina principalmente a satisfacer la demanda final como el caso de "otros cereales", "abastecimiento de agua", "servicios de administración pública", "servicios médicos y de salud"; finalmente 10 se clasifican como "No Manufactureras/Destino intermedio" Su demanda por insumos es baja, estos productos básicamente sirven de insumo a otras actividades y muy poco de ellos son entregados en el mercado como producto final. Este es el caso de "otros servicios", "comercio al por mayor y al detalle", "silvicultura", "bienes raíces, entre otros".

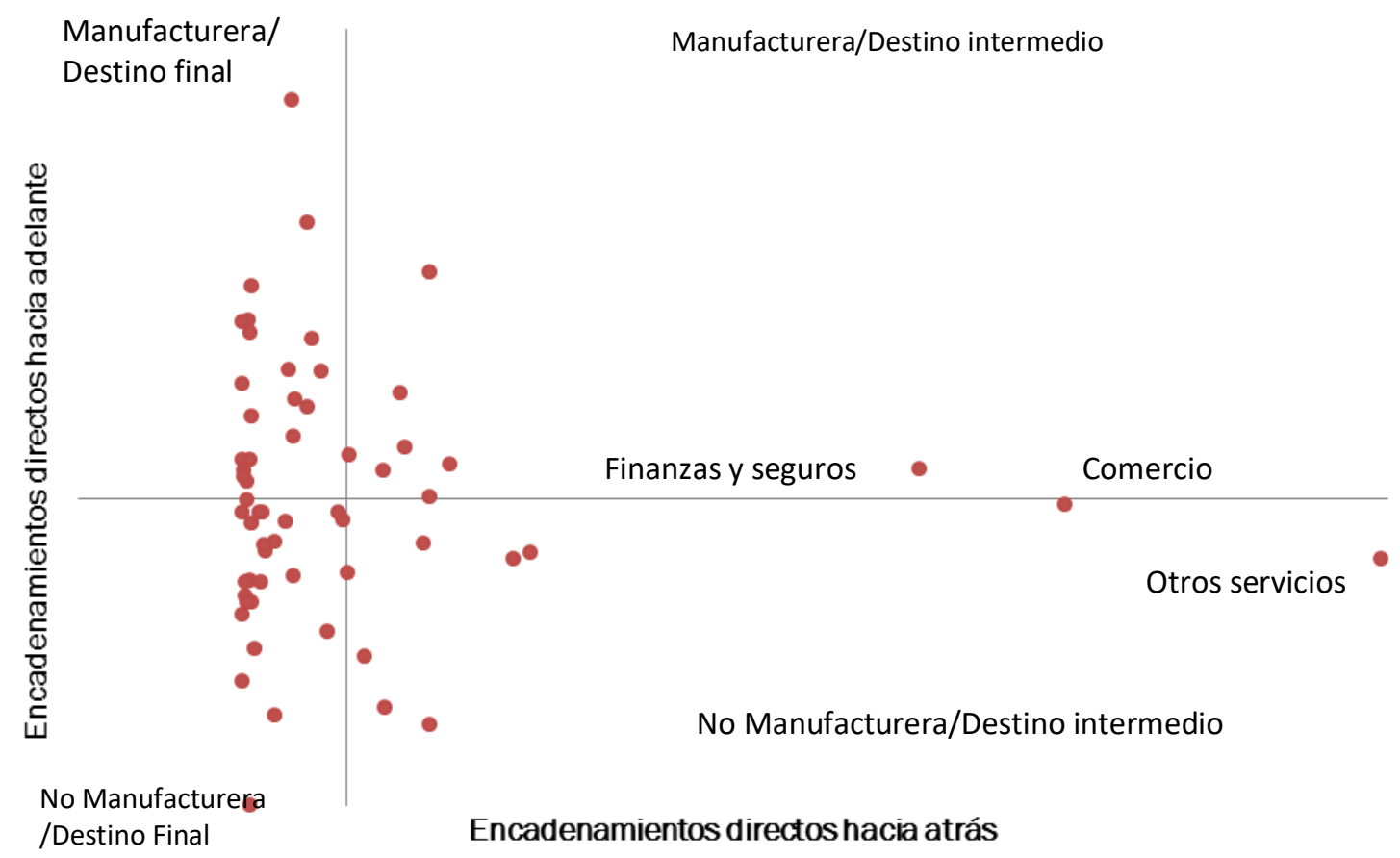

Figura 8. Actividades y clasificación. Fuente: elaboración propia. 


\section{Efectos multiplicadores}

De las secciones anteriores se puede concluir que el estudio de los encadenamientos permite analizar la estructura productiva, pero a partir de dicho análisis no se puede cuantificar los efectos multiplicadores que cada producto puede tener sobre otros productos.

De acuerdo con Hernández (2012) un multiplicador es un coeficiente numérico que indica la magnitud del cambio de una variable (generalmente endógena) producto de una variación de una variable que ha cambiado. De forma más puntual el multiplicador refleja la "magnitud" de la variación de la variable endógena ante cambios en la variable exógena.

En el contexto de la MIP el multiplicador del producto muestra el efecto multiplicador total que tiene la producción de una actividad sobre la producción de todas las actividades. Este indicador contiene dos tipos de efectos: los directos y los indirectos. Los primeros miden la capacidad de una actividad de arrastrar directamente a otras ligadas a ella, por su demanda de bienes de consumo intermedio. Es decir, se concentran en las relaciones entre actividades en una primera instancia, sin tener en cuenta las sucesivas rondas de compras intermedias.

Por otro lado, el efecto indirecto mide la capacidad de una actividad productiva de arrastrar indirectamente a otras ligadas a ella, por su demanda de bienes de consumo intermedio. Miden por lo tanto los efectos adicionales que se producen sobre la demanda de insumos de otras actividades, luego de una primera vuelta de compras intermedias.

Estos multiplicadores ( $\mathrm{BL}$ y $\mathrm{FL}$ ) se estiman tanto para los encadenamientos hacia atrás como para los encadenamientos hacia adelante de la siguiente forma:

$$
\text { (1.6) } B L_{j}=\sum_{i} b_{i j}
$$

Y para los encadenamientos hacia adelante:

$$
\text { (1.7)FL } L_{j}=\sum_{i} b_{i j}
$$

Dado lo anterior y con la información disponible es posible identificar que, de los productos con mayores encadenamientos hacia atrás, los "productos cárnicos y productos lácteos" tienen el mayor multiplicador del producto $(2,13)$, seguido de "granos y harina molida" $(1,84)$ y "otras construcciones" $(1,75)$. De los anteriores, los mayores efectos indirectos son de los "productos cárnicos y productos lácteos" $(1,06)$. Esto sugiere que ante un cambio de 1 unidad en la

20

Carlos Chaverri Morales

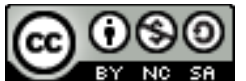

Revista Economía y Sociedad by Universidad Nacional is licensed under a Creative Commons Reconocimiento-NoComercial- 
demanda de Productos cárnicos y productos lácteos el efecto total sobre la producción será de 2,13 unidades, de las cuales 1,07 obedecen a efectos directos y 1,06 a efectos indirectos.

El "vidrio y los productos de vidrio" tienen los mayores efectos indirectos $(1,17)$. Como era de esperar el caso del "comercio al por mayor y al detalle" y los "otros servicios" tiene bajos efectos indirectos en comparación con los previamente mencionados, 0,41 y 0,22 respectivamente.

Al analizar los productos con mayores encadenamientos hacia adelante se puede constatar que los "otros servicios", "comercio al por mayor y al detalle", "finanzas, seguros y el transporte" no solo son actividades con altos encadenamientos hacia adelante, sino que además tiene altos multiplicadores. En el caso de primer grupo tiene un multiplicador del producto de 6,75; un bajo efecto multiplicador directo $(1,13)$ y un alto efecto indirecto $(5,62)$. Esta característica es común a los otros productos que se mencionan en el párrafo previo tal y como se muestra en la tabla 6 y refuerza la idea de que estos productos influyen fuertemente de forma indirecta sobre la demanda de insumos de otros.

Tabla 6.

\section{Multiplicador del producto directo e indirecto}

\begin{tabular}{|c|c|c|c|c|c|c|c|c|}
\hline & & & Demand & & & & Oferta & \\
\hline & & Total & Directos & Indirectos & & Total & Directos & Indirectos \\
\hline 1 & $\begin{array}{l}\text { Productos cárnicos y } \\
\text { productos lácteos }\end{array}$ & 2,13 & 1,07 & 1,06 & Otros servicios & 6,75 & 1,13 & 5,62 \\
\hline 2 & Granos y harina molida & 1,84 & 1,00 & 0,84 & Finanzas y seguros & 4,32 & 1,20 & 3,13 \\
\hline 3 & $\begin{array}{l}\text { Otros productos } \\
\text { alimenticios }\end{array}$ & 1,79 & 1,07 & 0,72 & $\begin{array}{l}\text { Comercio al por mayor } \\
\text { y a detalle }\end{array}$ & 4,19 & 1,01 & 3,18 \\
\hline 4 & Otras construcciones & 1,75 & 1,00 & 0,75 & Transporte & 2,22 & 1,03 & 1,19 \\
\hline 5 & Productos pesqueros & 1,75 & 1,00 & 0,75 & Electricidad y gas & 2,14 & 1,01 & 1,13 \\
\hline 6 & Madera & 1,75 & 1,04 & 0,70 & Bienes raíces & 2,05 & 1,01 & 1,04 \\
\hline 7 & $\begin{array}{l}\text { Vidrio y productos de } \\
\text { vidrio }\end{array}$ & 1,72 & 1,17 & 0,55 & $\begin{array}{l}\text { Otros productos } \\
\text { alimenticios }\end{array}$ & 1,83 & 1,07 & 0,76 \\
\hline 8 & Hoteles & 1,70 & 1,00 & 0,70 & $\begin{array}{l}\text { Construcción de } \\
\text { edificios }\end{array}$ & 1,81 & 1,06 & 0,75 \\
\hline 9 & Tabaco & 1,66 & 1,16 & 0,50 & $\begin{array}{l}\text { Fertilizantes y } \\
\text { pesticidas químicos }\end{array}$ & 1,72 & 1,07 & 0,64 \\
\hline 10 & Restaurantes & 1,66 & 1,00 & 0,65 & Papel y pulpa de papel & 1,70 & 1,22 & 0,48 \\
\hline
\end{tabular}

Fuente: elaboración propia.

Adicionalmente, se lleva a cabo un ejercicio de simulación para estimar el número de empleos directos e indirectos que se generan en los productos del sector servicios a partir de un choque 
exógeno en la demanda final por un millón de dólares. Para ello, se estima un multiplicador de empleo que se obtiene a partir de un modelo cerrado de insumo producto, es decir, que incluye a los hogares como un sector industrial. El multiplicador se obtiene como el cociente de requerimientos técnicos directos e indirectos sobre el coeficiente de empleo directo.

Dicha estimación permite obtener los siguientes resultados: para el caso del "comercio al por mayor y al detalle" se obtiene la generación de 62 empleos directos y 7 empleos indirectos; "hoteles" 36 empleos directos y 17 empleos indirectos y el caso de "restaurantes" 29 empleos directos y 19 empleos indirectos.

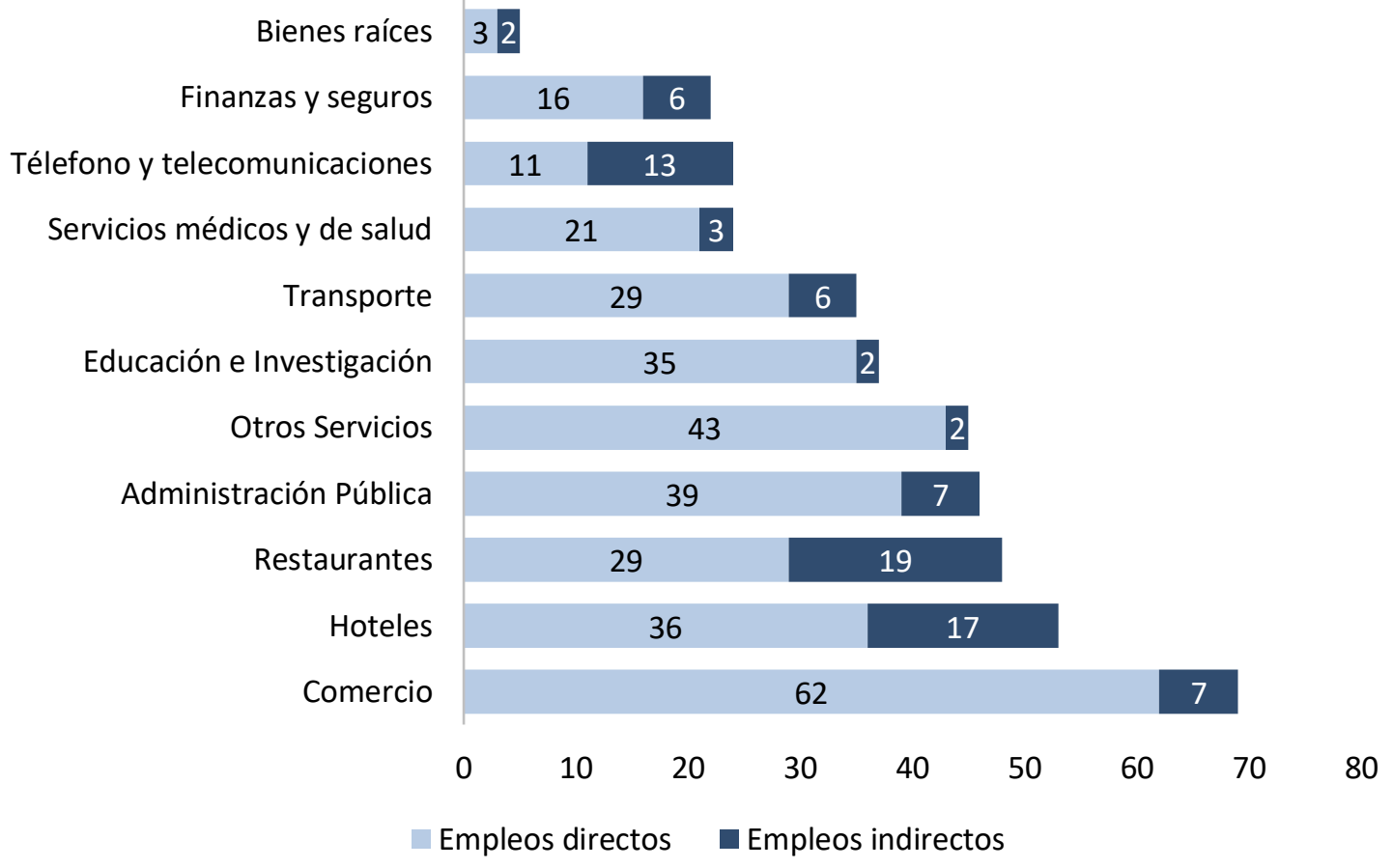

Figura 9. Incrementos en el número de empleo directo e indirecto ante un choque de demanda final. Sector servicios. Fuente: elaboración propia.

\section{Conclusiones}

El presente trabajo constituye un primer análisis descriptivo de las principales características de la estructura productiva costarricense según la información de la MIP-2011. Dado que no se dispone de información más reciente, no se pueden probar otras hipótesis relacionadas con posibles cambios estructurales y cambio en los valores de los encadenamientos y los multiplicadores en periodos más recientes. En la medida en que se disponga de matrices de insumo producto con mayor frecuencia, las posibilidades de análisis técnico aumentan y con ello la calidad de la información para la toma de decisiones.

22 
Es claro que identificar en el periodo de referencia cuáles son las actividades que más promueven el empleo y el crecimiento económico permite la definición de estrategias de mediano y largo plazo. Esto permite identificar las acciones necesarias para que se cumpla el objetivo de propiciar un ambiente adecuado para la articulación de los diferentes actores que están vinculados con la actividad productiva del país.

Con la información disponible se ha comprobado que los productos del sector terciario (servicios) presentan altos encadenamientos hacia adelante y bajos encadenamientos hacia atrás, esto constituye una fuente de información valiosa para el diseño de política económica dado que es un sector que año con año registra un alto número de nuevas empresas para la prestación de servicios, además dada la dinámica de crecimiento que registra en los últimos años es un sector que atrae mucho empleo y que en términos de ingresos salariales promedio, es mejor remunerado que otros.

Por ejemplo, en materia de empleo la MIP-2011 permite identificar que productos con mayor personal ocupado son los "otros servicios", el "comercio al por mayor y al detalle" y los "cultivos alimentarios". La ocupación en la elaboración de estos productos básicamente se concentra en personal no calificado.

Estos productos son los que poseen los mayores encadenamientos hacia adelante y además son las que tienen los mayores efectos multiplicadores sobre la producción. Esto sin duda permite tener un panorama más claro a la hora de examinar y diseñar políticas públicas tendientes a la promoción del empleo y el crecimiento.

\section{Bibliografía}

Arias y Sánchez. (2009). Análisis de la dinámica regional del empleo utilizando el modelo Shift Share especialmente modificado: el caso de la región chorotega, 1990-2009. Revista de Ciencias Económicas, 29(2), 1-20. Recuperado de http://revistas.ucr.ac.cr/index.php/economicas/article/view/7028

Bulmer-Thomas (1976). An Input-Output Planning Model for Costa Rica (Tesis Doctoral) Universidad de Oxford. Recuperado de https://www.researchgate.net/publication/35699241 An inputoutput planning model for Costa Rica

Chenery \& Watanabe, (1958). An International Comparison of the Structure of Production. Econometrica, 26(4), 487-521. Recuperado de http://dx.doi.org/10.2307/1907514

Claus (2003). Changes in New Zealand's Production Structure: An Input Output Analysis. New Zealand Treasury. Recuperado de http://www.treasury.govt.nz/publications/researchpolicy/wp/2003/03-01 
Gereffi, G; Humphrey, J; \& Sturgeon, T. (2005). The governance of global value chains. Review of International Political Economy, 12(1), 78-104. Recuperado de http://dx.doi.org/10.1080/09692290500049805

Hernández, G. (2012). Matrices de insumo producto y análisis de multiplicadores: una aplicación para Colombia. Revista de Economía Institucional, 14(26), 1-19. Recuperado de http://www.economiainstitucional.com/esp/num_ant/rei26.htm

Hirschman, A. (1958). The Strategy of Economic Development. Recuperado de https://www.amazon.com/Strategy-Economic-Development-AlbertHirschman/dp/0300001177

Humphrey y Schmitz. (2002). How does insertion in global value chains affect upgrading in industrial clusters? Regional Studies, 36(9), 1017-1027. Recuperado de http://www.tandfonline.com/doi/abs/10.1080/0034340022000022198?journalCode=cr es20\#.VOyohTXhC1s

Instituto Nacional de Estadística e Informática. (2001). Multiplicadores de Economía Peruana una aplicación de la tabla Insumo-Producto 1994 (Colección cambio de año base de las cuentas nacionales del Perú, Documento 6). Recuperado de https://www.inei.gob.pe/media/MenuRecursivo/publicaciones digitales/Est/Lib0469/Li bro.pdf

Kula (2008). Supply -Use and input-output tables, backward and forward linkages of the Turkish economy. Recuperado de http://ma.umd.edu/papers/conferences/2008/kula.pdf

Leiva y Vargas (1972). Modelo Insumo-Producto para Costa Rica-1969: Un ensayo de Economía Inter-industrial (Tesis Licenciatura). Universidad de Costa Rica, San Pedro de Montes de Oca, Costa Rica.

Miller \& Blair. (2009). Input-Ouput Analysis: Foundations and Extensions. Recuperado de https://books.google.co.cr/books/about/Input_Output_Analysis.html?id=SmFUl6X1FUC\&redir esc=y

Naciones Unidas. (2008). Sistema de Cuentas Nacionales 2008. Recuperado de http://unstats.un.org/unsd/nationalaccount/docs/SNA2008Spanish.pdf

Rasmussen, P. (1958). Studies in Inter-Sectoral Relations, Amsterdam, North- Holland P. C. Recuperado de https://books.google.co.cr/books/about/Studies in inter sectoral relations.html?id=n0 OsAAAAMAAJ\&redir esc=y

24

Carlos Chaverri Morales

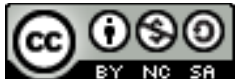

Revista Economía y Sociedad by Universidad Nacional is licensed under a Creative Commons Reconocimiento-NoComercial- 
Robles y Polanco (2011). "Encadenamientos hacia atrás y hacia adelante en la economía del Valle del Cauca año 2004". Tesis de Grado Meritorio de la Maestría en Economía de la Universidad Autónoma de Occidente Cali, Colombia. Recuperado de http://dali.uao.edu.co:7777/pls/portal/url/ITEM/2EEOF2D2F90BE750E04010AC20C97C7 $\underline{4}$

Sánchez (2006). "Matriz de contabilidad social (MCS) 2002 de Costa Rica, y los fundamentos metodológicos de su construcción." Serie Estudios y Perspectivas, № 47. México D.F. Recuperado de http://repositorio.cepal.org/bitstream/handle/11362/4977/1/S2006603 es.pdf

Schuschny, A. (2005). Tópicos sobre el Modelo de Insumo-Producto: teoría y aplicaciones. Recuperado del sitio web de la comisión Económica para América Latina y el Caribe: http://www.cepal.org/deype/noticias/noticias/0/22350/redima2005 schuschny.pdf

\section{Anexo 1}

\section{Actividades dentro del grupo "Otros servicios prestados a empresas"}

- Servicios administrativos y de apoyo de oficina y otras actividades de apoyo a las empresas. Servicios de alquiler de automotores, maquinaria y equipo.

- Servicios de publicidad, provisión de espacios de publicidad y estudios de mercado.

- Servicios de consultoría en gestión financiera, recursos humanos, comercialización, oficinas principales y afines.

- Servicios de información, programación y consultoría informática, excepto edición de programas informáticos y afines.

- Servicios domésticos.

- Servicios de agencias de viajes, operadores turísticos.

- Servicios de reservas y actividades conexas.

- Otros servicios profesionales, científicos y técnicos.

- Servicios de reparación e instalación de maquinaria y equipo.

- Servicios de seguridad e investigación.

- Servicios artísticos, de entretenimiento y recreativos.

- Limpieza de edificios y cuidado del paisaje y mantenimiento.

- Servicios de arquitectura, ingeniería y conexos.

- Otros servicios. 